Bull. Chem. Soc. Ethiop. 2013, 27(3), 447-457.

Printed in Ethiopia

ISSN 1011-3924

DOI: http://dx.doi.org/10.4314/bcse.v27i3.14

(c) 2013 Chemical Society of Ethiopia

\title{
SYNTHESIS, RHEOLOGICAL BEHAVIOR AND SWELLING PROPERTIES OF COPOLYMER HYDROGELS BASED ON POLY(N-ISOPROPYLACRYLAMIDE) WITH HYDROPHILIC MONOMERS
}

\author{
N. Seddiki* and D. Aliouche \\ Laboratory of Polymers Treatment and Forming, F.S.I., M'Hamed Bougara University, \\ Boumerdes, 35000, Algeria
}

(Received July 11, 2012; revised May 24, 2013)

\begin{abstract}
In this study, hydrogels of poly(N-isopropylacrylamide-co-acrylamide) and poly(Nisopropylacrylamide-co-acrylic acid) having a thermoresponsive character were prepared by aqueous free-radical co-polymerization using the ammonium persulfate/N,N,N', N'-tetramethylethylenediamine (APS/TEMED) redoxpair initiator system in the presence of N,N'-methylenebisacrylamide (MBAAm) crosslinker. (NIPAAm-co-AAm) and (NIPAAm-co-AAc) hydrogels with different thermoresponsive properties were obtained by fixing the initial NIPAAm/AAm mole ratio and and (NIPAAm-co-AAc) mole ratio to 80/20 and changing the crosslinker concentration. The copolymers were characterized with infrared spectroscopy (IR) and differential scanning calorimetry (DSC) techniques. The swelling response of the copolymers networks as a function of time, temperature and swelling environment has been observed to be dependent on both structural aspects of the polymers and swelling environment. The swelling has been observed to be decrease with increase in MBAAm in the copolypolymers networks. Rheological behavior was studies in oscillatory module. All copolymers have a viscoelastic behaviour. We note that the elastic modulus $G^{\prime}$ increases with increasing hydrophilic monomers.
\end{abstract}

KEY WORDS: Poly(N-isopropylacrylamide-co-acrylamide), Poly(N-isopropylacrylamide-co-acrylic acid), Thermoresponsive polymer, Swelling behavior, Rheological behavior

\section{INTRODUCTION}

Considerable research attention has been focused recently on materials that change their structure and properties in response to external chemical and/or physical stimuli [1-3]. These materials are called "intelligent" or "smart" materials. They are also named as "stimuliresponsive" or "enviromental" polymers. Stimuli-sensitive hydrogels are three-dimensional hydrophilic polymer network, that exhibit volume or phase transition in response to external envirommental changes, such as temperature, $\mathrm{pH}$, strength ionic, pressure electronic and magnetic field. They are widely used in many applications such as hygiene, cosmetic, agriculture, medicine, biotechnologie [4-5], including controlled drug release [6, 7] in separation processus [8], and for the immobilization of enzymes [9] in textiles to improve or achieve textile smart functionalities [10].

Poly(N-isopropylacrylamide) (PNIPAAm) gel is a typical thermosensitive polymer exhibiting the volume phase transition at approximately $33{ }^{\circ} \mathrm{C}$ (LCST: lower critical solution temperature) [11-12], below this temperature, the hydrogel is swollen, hydrated and hydrophilic, whereas above the LCST, the hydrogel shrinks and forms a collapsed, dehydrated and hydrophobic state due to the breakdown of the delicate hydrophilic/hydrophobic balance in the network structure [13]. This distinctive property of PNIPAAm is attributed to its unique rapid alternation in hydrophilicity and hydrophobicity around LCST [14]. The hydrophilic groups (CONH-) of PNIPAAm hydrate to form an expanded structure when the temperature is below LCST. However, as the PNIPAAm hydrogel is heated above LCST, the polymer chains collapse

*Corresponding author. E-mail: n_Seddiki1@yahoo.fr 
abruptly and phase separation occurs. Such a unique phase transition of the PNIPAAm hydrogel upon external temperature changes has been investigated widely [15].

The LCST can be controlled by copolymerization with other monomers [16]. The addition of hydrophilic monomers generally increases the LCST whereas the incorporation of more hydrophobic units/groups has the opposite effect. The swelling degree of hydrogels depends not only on the nature of hydrogel and swelling medium, but also on the crosslink density. Many studies have been reported on thermosensitive hydrogels based on the monomer NIPAAm and its copolymers with most widely studied comonomer, such as acrylic acid, acrylamide [17-18] methyl-acrylamido propanesulfonic acid, sodium acrylate, sodium methacrylate [19], itaconic acid, maleic acid, $\mathrm{N}$-isopropyl-maleamic acid [20-21].

The present work focuses on the synthesis of PNIPAAm and copolymer hydrogel based on acrylamide (AAm) and acrylic acid (AAc) with changing concentration of N,N'methylenebisacrylamide (MBAAm), as the crosslinker. The swelling properties of the hydrogels, in water at different temperature, were systematically studied, and the effect of crosslinker contents in the swelling and behavior rheological has been noticed.

\section{EXPERIMENTAL}

\section{Materials}

N-Isopropylacrylamide (NIPAAm), acrylamide (AAm) and acrylic acid (AAc) monomers used in this study were obtained from Aldrich Chemical Co. Acrylamide was purified by recrystallization from methanol and dried in vacuum desiccator over silica gel; acrylic acid was purified by vacuum distillation.

The crosslinker N,N'-methylenebisacrylamide (MBAAm), the activator N,N,N',N'tetramethyl ethylene diamine (TEMED) and the initiator ammonium peroxodisulphate (APS) were all analytical grade and purchased from Panreac Monplet \& Esteban, S.A. Spain. All reagents were used without further purification. Deionized water was used throughout the experiments in the preparation of hydrogels and in the swelling experiments.

Methods

Synthesis of hydrogels

Hydrogels were prepared by free radical crosslinking copolymerization in deionized water, which is a solvent for all components of the feed mixture. First, the PNIPAAm monomer was used as a base monomer in the synthesis of hydrogel copolymers; the comonomers are AAc carrying acid group and AAm. Aqueous solutions of PNIPAAm were prepared in deionized water. Different amounts of crosslinker agent were added to copolymers: PNIPAAm-co-AAc and PNIPAAm-co-AAm solution (mole ratios: 80/20). Table 1 describes the different formulations used in this work. After nitrogen bubbling for $15 \mathrm{~min}$, free radical copolymerization of hydrogels was carried in glass tubes of $10 \mathrm{~mm}$ inner diameter at room temperature for $2 \mathrm{~h}$, using APS and TEMED as redox initiator. Subsequently, the crosslinking of PNIPAAm hydrogels was carried out by MBAAm at various concentrations $(0.25,0.5$ and $1 \% \mathrm{MBAAm} /$ weight (total monomer + crosslinker)). After polymerization, crosslinked copolymers were removed from tubes and the hydrogels obtained in long cylindrical shapes were cut into pieces of approximately $1 \mathrm{~cm}$ length. Polymer samples were immersed in distilled water at room temperature for $48 \mathrm{~h}$ and the water was refreshed every several hours in order to allow the unreacted chemicals to leach out. Finally extracted gels were dried in a vacuum oven at $30^{\circ} \mathrm{C}[22]$. 
Table 1. Compositions of the PNIPAAm-co-AAc and PNIPAAm-co-AAm copolymer hydrogels.

\begin{tabular}{|l|c|c|c|c|c|c|}
\hline Sample & NIPAAm $(\mathrm{g})$ & AAm $(\mathrm{g})$ & AAc $(\mathrm{mL})$ & MBAAm $(\%)$ & TEMED $(\mathrm{mL})$ & APS $(\mathrm{mL})$ \\
\hline PNIPAAm (100\%) & 1 & - & - & 0.5 & 2.5 & 2.5 \\
PNIPAAm-co-AAm (80/20)\% & 0.5 & 0.078 & - & 0.25 & 2.5 & 2.5 \\
& 0.5 & 0.078 & - & 0.5 & 2.5 & 2.5 \\
PNIPAAm-co-AAc (80/20)\% & 0.5 & 0.078 & - & 1 & 2.5 & 2.5 \\
& 0.5 & - & 0.06 & 0.25 & 2.5 & 2.5 \\
& 0.5 & - & 0.06 & 0.5 & 2.5 & 2.5 \\
& 0.5 & - & 0.06 & 1 & 2.5 & 2.5 \\
\hline
\end{tabular}

\section{Infrared spectroscopy}

In infrared spectroscopic analysis, measurements were recorded in a $\mathrm{KBr}$ phase by using a Shimadzu M850 Model FT-IR Spectrophotometer with $20 \mathrm{scans}$ and $2 \mathrm{~cm}^{-1}$ resolution for samples of hydrogel copolymers. All samples were analyzed in the range $4000-400 \mathrm{~cm}^{-1}$.

\section{Differential scanning calorimetry (DSC)}

Thermal analysis was performed using a NETZSCH DSC 204 F1 Phoenix thermal apparatus, under nitrogen flow. The samples were heated in an aluminum holder from room temperature to $250{ }^{\circ} \mathrm{C}$ at a rate of $20{ }^{\circ} \mathrm{C} \mathrm{min}{ }^{-1}$, on $5 \mathrm{mg}$ sample. The heat flow (DSC curve) was recorded simultaneously as a function of temperature.

\section{Rheological measurements}

The rheological measurements of aqueous suspensions of PNIPAAm, PNIPAAm-co-AAm and PNIPAAm-co-AAc copolymers were carried out in controlled stress AR-2000 Advanced Rheometer (T. A. Instruments) under the oscillatory shear. The flow of dilute hydrogel suspensions under shear was studied in Couette geometry, using a pair of coaxial cylinders with $15 \mathrm{~mm}$ of external diameter and gap of $1 \mathrm{~mm}$ [23]. Silicone oil was added to the surface of the sample to prevent evaporation during measurements.

The suspensions of gel particles swollen to different extent were prepared by simply mixing dry gel powder with a certain amount of deionized water. The size of the dry gel particles is about 0.1-0.2 $\mathrm{mm}$ with a maximal degree of swelling in water of about $250 \mathrm{~g} / \mathrm{g}$.

The rheological behavior of polymer suspensions of $0.1 \mathrm{wt} \%$ concentrations was studied at room temperature. In oscillatory mode, the experiments were done at $6.2 \mathrm{~Hz}$. The gel properties were described in terms of two dynamic mechanical properties: the elastic modulus G' (also known as dynamic rigidity), reflecting the reversibly stored energy of the system, and the viscous modulus G', reflecting the irreversible energy loss.

\section{Swelling measurements}

Swelling measurements: For several applications, in particular in the medical and pharmaceutical fields, the swelling behavior of the gels was investigated in deionized water. The effect of temperature of the swelling medium on the equilibrium water uptake of the hydrogel samples was determined at different temperature $\left(25-70{ }^{\circ} \mathrm{C}\right)$ and removed from water at regular time intervals.

The swollen gels were taken out at regular time-intervals, wiped superficially with filter paper to remove surface-bound water, weighed and then placed in the same bath. The percent mass swelling was determined using the following expression: 
$\%$ Swelling $=\frac{\mathrm{M}_{\mathrm{t}}-\mathrm{M}_{\mathrm{o}}}{\mathrm{M}_{\mathrm{o}}} \times 100$

where $\mathrm{M}_{0}$ and $\mathrm{M}_{\mathrm{t}}$ are the initial mass and mass at different time-intervals, respectively. All the experiments were carried out in triplicate and the average values have been reported in the data.

\section{RESULTS AND DISCUSSION}

\section{Infrared spectroscopy}

Figure 1 presents the FTIR spectra of PNIPAAm hydrogel, A broad bands appeared between 3600 and $3200 \mathrm{~cm}^{-1}$ (N-H stretching), in PNIPAAm and MBAAm, at $3075 \mathrm{~cm}^{-1}$ the stretching frequency of amide. Peaks at $1654 \mathrm{~cm}^{-1}$ and $1545 \mathrm{~cm}^{-1}$, which is due to the presence of the amide groups (amide band I and II, respectively) in NIPAAm and MBAAm, and $1380 \mathrm{~cm}^{-1}$ corresponding to the $\mathrm{C}-\mathrm{H}$ vibrations of $-\mathrm{CH}\left(\mathrm{CH}_{3}\right)_{2}$ in the PNIPAAm [24-25].

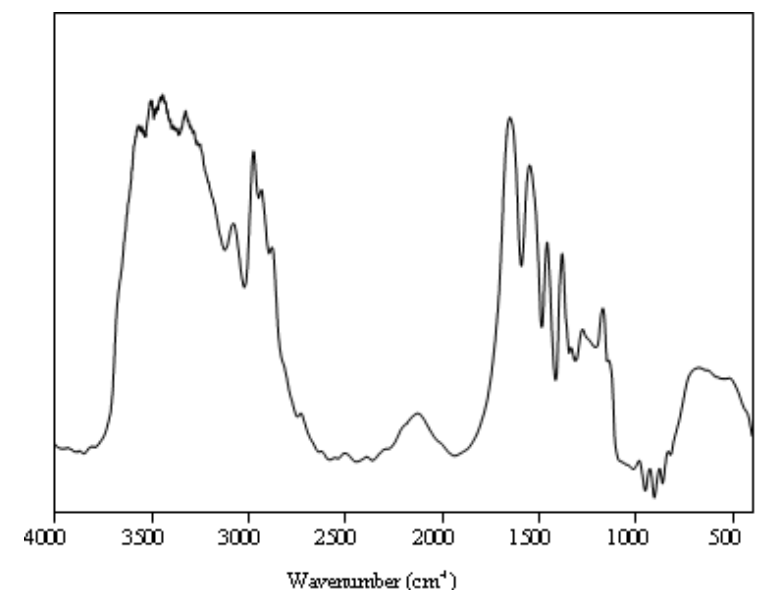

Figure 1. FT-IR spectra of the PNIPAAm).

The FTIR spectra of PNIPAAm (a), PNIPAAm-co-AAc (b) and PNIPAAm-co-AAm (c) hydrogels with 80/20 \% ratio are shown in Figure 2. Asymmetrical and symmetrical stretching of $\mathrm{C}-\mathrm{H}$ is found at 2900 and $2740 \mathrm{~cm}^{-1}$, respectively, carbonyl stretching vibration $\mathrm{C}=\mathrm{O}$ to the carboxyl group gives absorption peak at $1720 \mathrm{~cm}^{-1}$ of acrylic acid. The contribution of AAm confirmed at $1424 \mathrm{~cm}^{-1}$ which is due to the presence of amide groups of polyacrylamide. The broad band at $1640 \mathrm{~cm}^{-1}$ can be assigned to the overlapping of NIPAAm-co-AAc and NIPAAmco-AAm, the presence of these bands in the FTIR spectra of the PNIPAAm-co-AAc and PNIPAAm-co-AAm hydrogels confirms the copolymerization reaction between NIPAAm and the monomers.

\section{Differential scanning calorimetry (DSC)}

The DSC of PNIPAAm and its copolymers PNIPAAm-co-AAc and PNIPAAm-co-AAm are shown in Figure 3. The measurement of the glass transition temperature $\left(\mathrm{T}_{\mathrm{g}}\right)$ of a hydrogel is often used as a criterion to determine its miscibility. The miscible hydrogel would exhibit a 
single transition between $\mathrm{T}_{\mathrm{g}}$ of the two components. The glass transition temperature of linear poly ( $\mathrm{N}$-isopropylacrylamide) homopolymer is between 85 and $130{ }^{\circ} \mathrm{C}$, probably depending on molecular weight [23]. In Figure 3(a), a relaxation endotherm at $122{ }^{\circ} \mathrm{C}$ identifies the glass transition temperature observed for the PNIPAAm [26]. The gel transition temperatures are increased with addition of the monomer AAc in the copolymeric composition. The DSC thermgrams corresponding to this sample is reported in Figure 3(b), that the presence of a single transition corresponding to the glass transition at $130{ }^{\circ} \mathrm{C}$ of $0.5 \%$ of MBAAm in the hydrogels is the result of interactions between the two polymers [27]. Similarly, the curve corresponding to PNIPAAm-co-AAm hydrogel shows a single thermal phenomenon, corresponding to the glass transition, which occurs at $127{ }^{\circ} \mathrm{C}$ for $0.5 \%$ of MBAAm (Figure 3c). In the case of the hydrogels, the single $\mathrm{T}_{\mathrm{g}}$ for all sample suggests that the hydrogels has good miscibility.

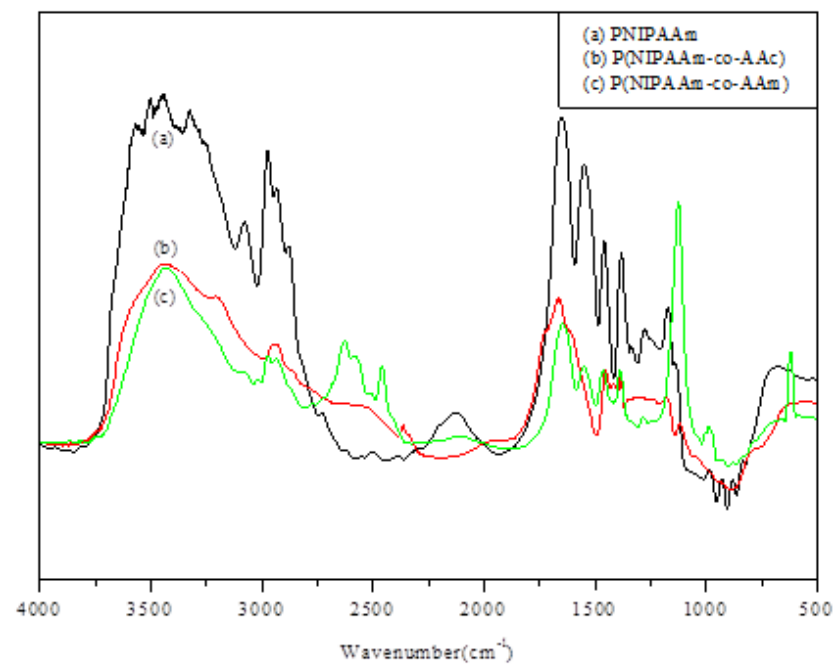

Figure 2. FT-IR spectra of the PNIPAAm (a), PNIPAAm-co-AAc (b) and PNIPAAm-co-AAm (c) hydrogel samples.

Study of the swelling

Swelling of PNIPAAm and its copolymers has been studied by absorption of distilled water at different temperatures of $25{ }^{\circ} \mathrm{C}$ to $70{ }^{\circ} \mathrm{C}$, in a time interval of up to $350 \mathrm{~min}$. Figure 4 shows the swelling of PNIPAAm in water.

For the variation of the equilibrium-swelling ratio at medium temperature, the thermoresponsive PNIPAAm gel exhibited a sharp transition at $32{ }^{\circ} \mathrm{C}$ as was expected, in Figure 4 [28]. At a temperature below the LCST, a large expansion of the network leads to high absorption (presence of hydrophilic groups-CONH). At a temperature above the LCST increased system entropy occurs [29-30]. Swelling decreases due to hydrophobic interactions of hydrophobic groups of the hydrogels that are dominant. The maximum swelling is obtained at $25^{\circ} \mathrm{C}$. So below the LCST, the polymer swells, above the LCST it becomes opaque white, and collapses. 


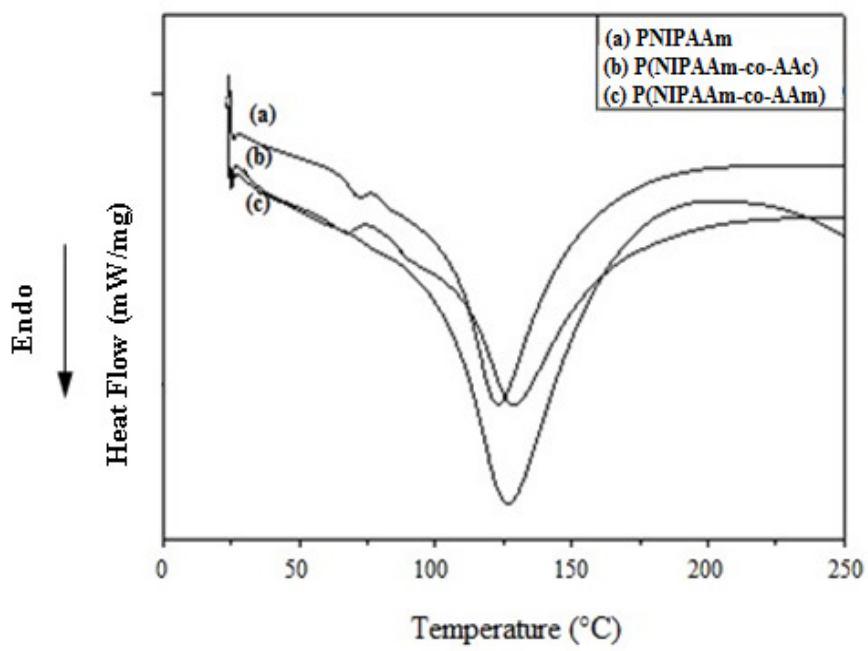

Figure 3. DSC thermograms of the PNIPAAm (a), PNIPAAm-co-AAc (80/20) (b) and PNIPAAm-co-AAm (80/20) (c) hydrogel samples.

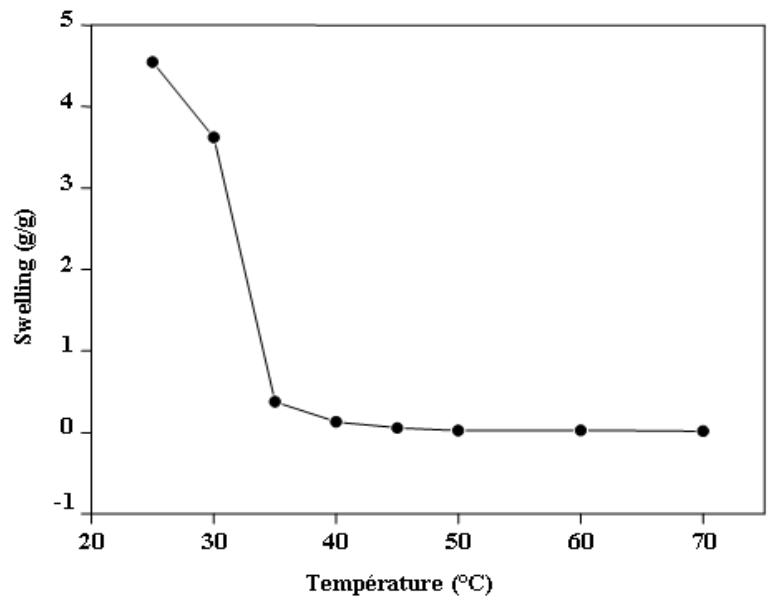

Figure 4. Influence of temperature on the water uptake of the poly ( $\mathrm{N}$-isopropylacrylamide) hydrogel.

A contribution of AAm plays a role on the value of the LCST as seen in Figure 8. The incorporation of AAm (which is hydrophilic) reduces the amount of hydrophobic groups and increases hydrophilité the polymer which leads to a strong interaction between water and hydrophilic groups of the polymer, and consequently a shift in the LCST to the right [31]. Therefore, the incorporation of hydrophilic or hydrophobic monomers in the gel composition can control the behavior of phase transition

In the copolymerization experiments, the MBAAm concentration was changed by fixing the initial NIPAAm/AAm mole ratio to $80 / 20$. The variation of the swelling ratio of the produced gels with medium temperature is given in Figure 5. 
As seen here, the swelling ratio of the copolymers decreased with increasing MBAAm concentration, which is due to the decrease of pore size [32]. The difference between the upper and lower plateau regions of the equilibrium-swelling curve decreased on increasing the MBAAm concentration. Increasing MBAAm quantity made the hydrogel more compact, so hydrogels with high mechanical strengths were obtained and this caused decreasing swelling capacities of these.
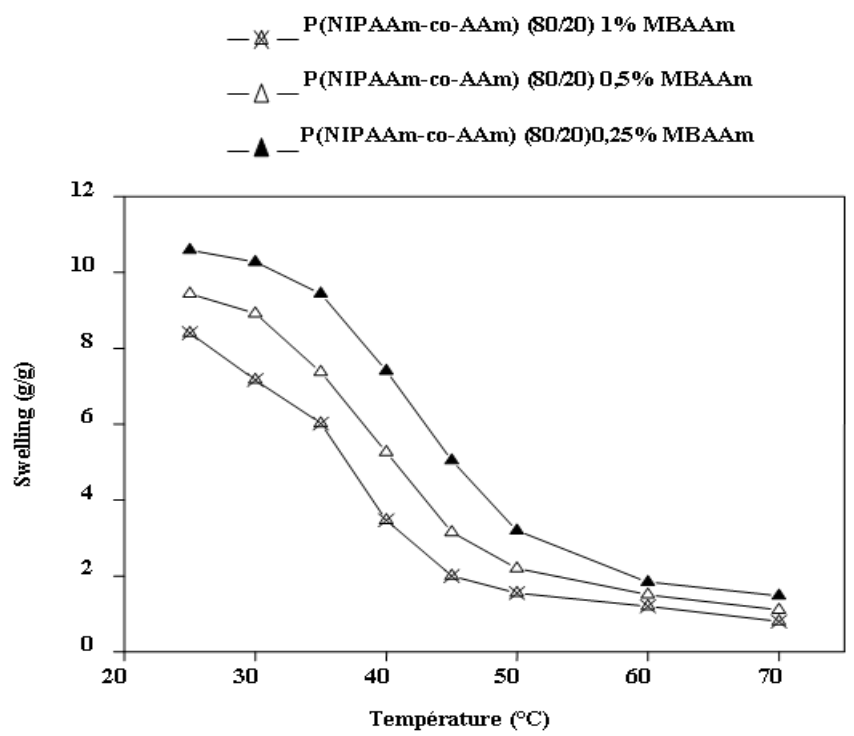

Figure 5. Influence of temperature and crosslinker agent (MBAAm) on the swelling of copolymers PNIPAAm-co-AAm hydrogel.

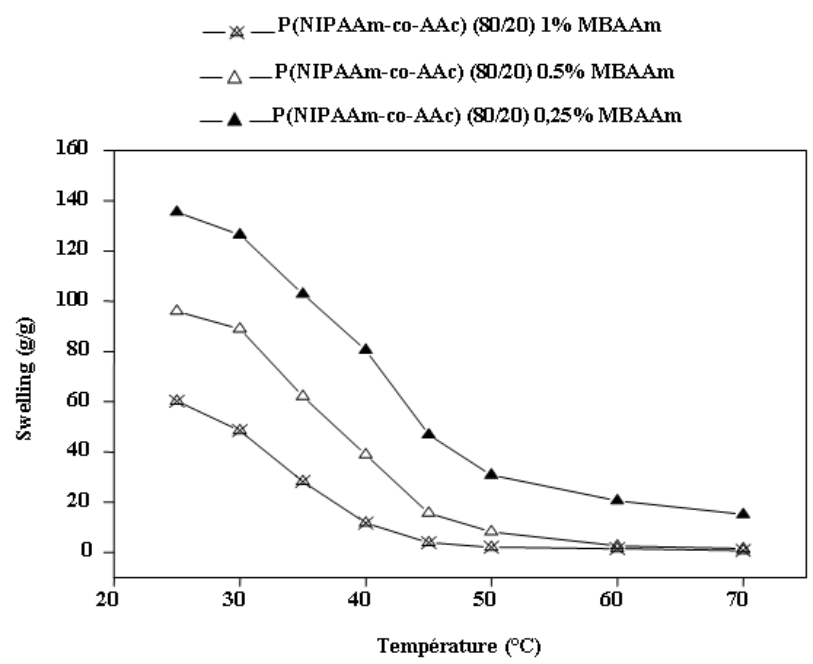

Figure 6. Influence of temperature and crosslinker agent (MBAAm) on the swelling of copolymers (NIPAAm-co-AAc) hydrogel.

Bull. Chem. Soc. Ethiop. 2013, 27(3) 
In Figure 6 we studied the influence of temperature and MBAAm on the swelling of copolymers of (NIPAAm-co-AAc). In this figure as seen the same behavior as before, that is to say, beyond a certain temperature the rate of swelling decreases sharply (above the LCST the network becomes more compact and rigid, the interpenetration of the water inside the network is very difficult), and the swelling is more important with the copolymers of acrylic acid copolymer that acrylamide increased swelling is due to the presence of- $\mathrm{COOH}$ groups increases electrostatic force (increased electrostatic repulsion between the carboxylate ions-COO-) [33], and the swelling is more important with decreasing concentration of crosslinker (MBAAm). This confirms previous results. We also note a shift of the LCST upward, and decrease the LCST when the concentration of MBAAm is important [34-35].We see from these results and the results obtained for AAm and NIPAAm, the largest swelling was observed for the copolymers of NIPAAm and AAc at low concentration of MBAAm, this can be explained by the presence of carboxylate- $\mathrm{COOH}$ groups of AAc that are more hydrophilic groups of - $\mathrm{CONH}_{2}$.

Therefore the incorporation of hydrophilic comonomers increases hydrophilite polymer (which is due to intercalation between water and hydrophilic groups), which consequently moves to the right LSCT otherwise hydrophilic interactions increase with increasing temperature which increasing polymer-water interactions [36]. However in both cases the LCST is less clear than for pure PNIPAAm. This temperature is spread over a larger interval.

\section{Study of rheological behavior}

Oscillatory rheometry provide information on the gel strength expressed as viscosity or elasticity and the relation of this strength with the gel composition and its stability. The gel properties were described in terms of two dynamic mechanical properties: the elastic modulus G' (also known as dynamic rigidity), reflecting the reversibly stored energy of the system, and the viscous modulus G", reflecting the irreversible energy loss. The effects of the copolymer composition on the elastic and viscous properties of the hydrogels are shown on Figures 7, 8 and 9. Here, G' and G" are shown as a function of the shear stress.

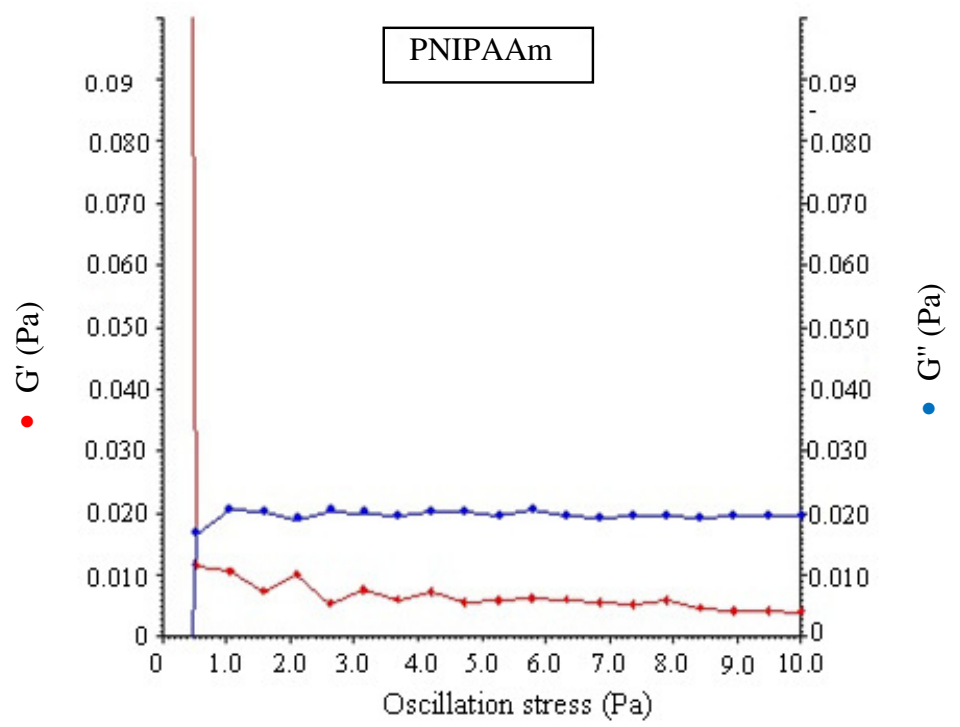

Figure 7. Evolution of the elastic modulus (G ') and viscous (G") of PNIPAAm.

Bull. Chem. Soc. Ethiop. 2013, 27(3) 


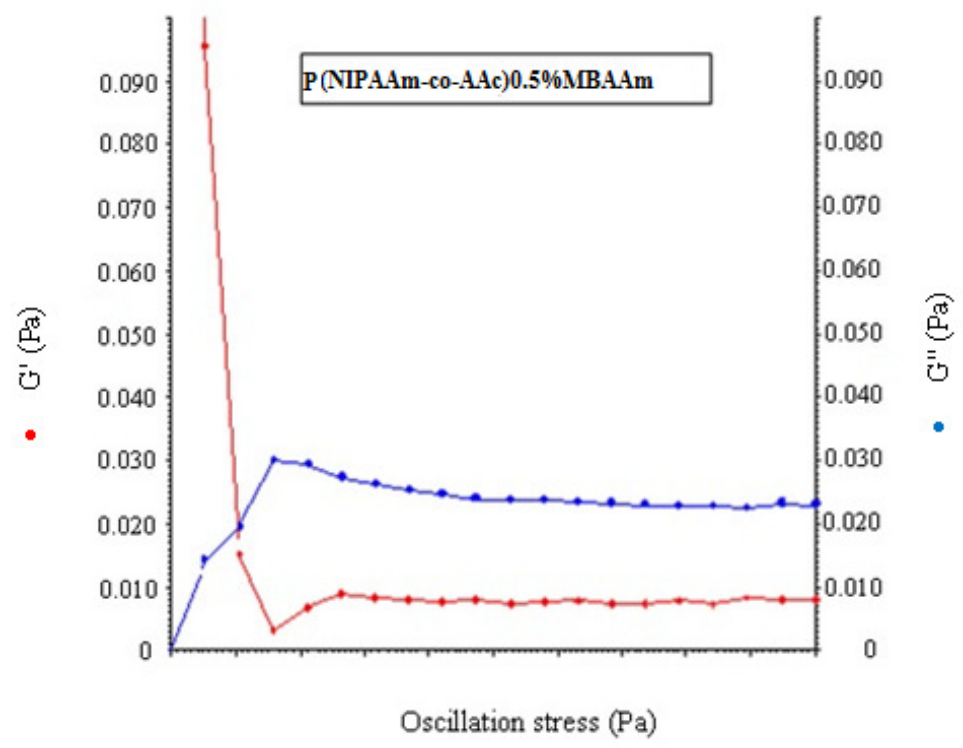

Figure 8. Evolution of the elastic modulus ( $\left.\mathrm{G}^{\prime}\right)$ and viscous $\left(\mathrm{G}^{\prime \prime}\right)$ of P (NIPAAm-co-AAc) $0.5 \%$ MBAAm.

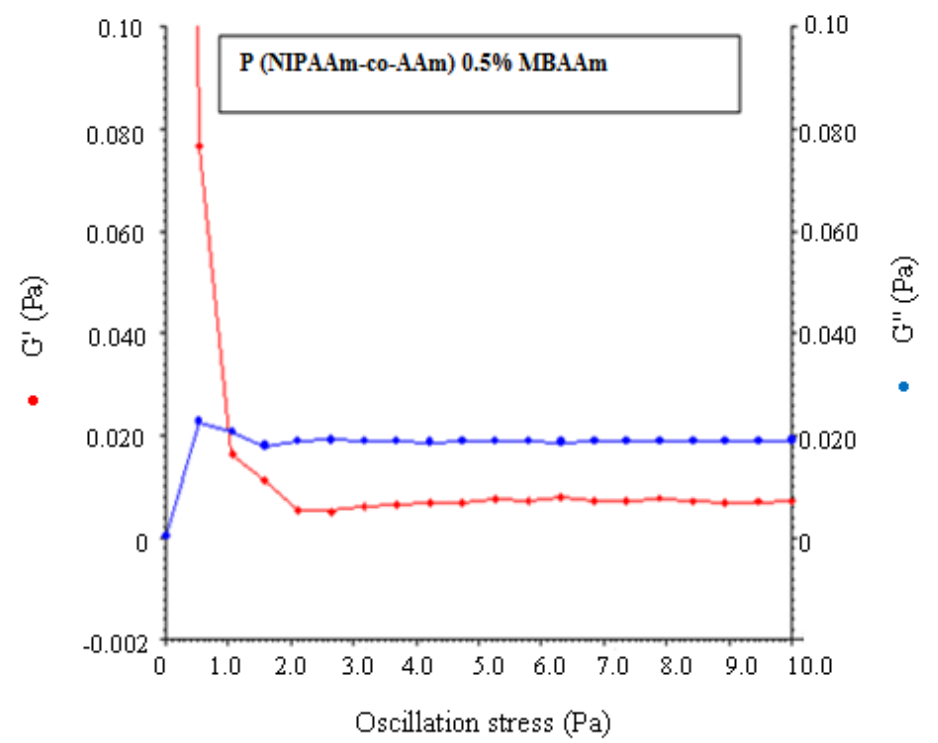

Figure 9. Evolution of the elastic modulus ( $\mathrm{G}^{\prime}$ ) and viscous $\left(\mathrm{G}^{\prime \prime}\right)$ of PNIPAAm-co-AAm 0.5\% MBAAm.

Bull. Chem. Soc. Ethiop. 2013, 27(3) 
The greatest values of $\mathrm{G}^{\prime}, \mathrm{G}^{\prime \prime}$ were associated with PNIPAAm-co-AAc, whereas the lowest observed values were observed with PNIPAAm. All copolymers have a viscoelastic behavior, we note that the elastic modulus $G^{\prime}$ increases with increasing acrylic acid concentration and acrylamide. In a first stage, a stress below $1 \mathrm{~Pa}$, so the elastic character dominates, so the increased rate of acrylic acid increases the number of elastic chains, which are held between two or more junction points formed by the intermolecular hydrophobic associations and the strength of the interactions involved in the second stage, a constraint than a $1 \mathrm{~Pa}, \mathrm{G}$ 'and G" evolve and remain stable over the long field stress in this plateau there is a loss of elasticity, which corresponds to the loss of intermolecular junctions nodes aware of the entanglement of chains under the effect of high stress, it is also possible that under the effect of shear too large, the polymer chains are broken, so the gel is unstructured.

\section{CONCLUSION}

PNIPAAm and its copolymers with hydrophilic monomers AAc and AAm were prepared through free radical polymerization using APS as initiator, TEMED as activator and BIS as crosslinking agent. The copolymer hydrogels were well characterized through FTIR, DSC, rheology and swelling measurements. The results indicated that the strong electrostatic interaction existed in the hydrogels, which resulted in the formation of a more stable co-polymer. In the DSC analyses, the single $\mathrm{T}_{\mathrm{g}}$ in the samples suggested that the two polymers in the hydrogel have good miscibility. The aqueous PNIPAAm-co-AAc and PNIPAAm-co-AAm polymer gel systems were investigated by dynamic rheometry. Viscoelastic properties have been investigated using oscillatory deformation tests. The PPNIPAAm-co-AAc hydrogels with addition concentration of AAc exhibited an enhancement in elastic modulus $G$ ' compared to the pure PNIPAAm hydrogel, indicating that adding AAc not only reinforced but also toughened PNIPAAm hydrogels, also AAm. Equilibrium swelling measurements were carried out in deionized water. The swelling of the hydrogels significantly increased with addition the hydrophilic monomers AAm and AAc concentrations in the gel matrix. The LCST increases when hydrophilic comonomers are incorporated into PNIPAAm. The swelling ratio increases with decreasing crosslinker MBAAm content, although the values of LCST are not affected by the crosslinker concentration. These gels can be used in applications such as immobilization of biologically active molecules and size-selective separations process.

\section{REFERENCES}

1. Zhang, X. D.; Wu, C.; Chu, C. Biomat. 2004, 25, 4719.

2. Soppimath, K.S.; Aminabhavi, T.M.; Dave, A.M.; Kumbar, S.G.; Rudzinski, W.E. Drug. Dev. Ind. Pharm. 2002, 28, 957.

3. Lee, S.B.; Ha, D.I.; Cho, S.K.; Lee, Y.M.; Kim, S.J. J. Appl. Polym. Sci. 2004, 92, 2612.

4. Song, H.; Zhang, S.; Ma, X.; Wang, D.J. Carbohyd. Polym. 2007, 69,189.

5. Emileh, A. ; Vasheghani-Farahani, E.; Imani, M. Eur. Polym. J. 2007, 43, 1986.

6. Gupta, P.; Vermani, K.; Garg, S. Drug. Disc. Today 2002, 10, 569.

7. Tas-delena, B.; Kayaman-Apohanb, N.; Guvenc, O.; Baysald, B.M. Rad. Phys. Chem. 2005, $73,340$.

8. Champ, S.; Xue, W.; Huglin, M.B. Polym. 2001, 42, 6439.

9. Dong, L.C.; Hoffman, A.S. J. Cont. Relea. 1986, 4, 223.

10. Jinlian, H.; Harper, M.; Guoqiang, Li.; Samuel, I.I. Smart. Mater. Struct. 2012, 21, 053001.

11. Heskins, M.; Guillet, J.E. Macromol. J. Chem. Sci. 1968, 2, 1441.

12. Chen, G.H.; Hoffman, A.S. Nature 1995, 373, 49.

Bull. Chem. Soc. Ethiop. 2013, 27(3) 
13. Hirokawa, Y.; Tanaka, T. J. Chem. Phys. 1984, 81, 6379.

14. Zhang, X.Z.; Yang, Y.Y.; Chung, T.S.; Ma, K.X. Langm. 2001, 17, 6094.

15. Kayaman, N.; Kazan, D.; Erarslan, A.; Okay, O.; Baysal, B.M. J. Appl. Polym. Sci. 1998, 67, 805.

16. Ruel-Gariepy, E.; Leroux, J.C. Eur. J. Pharm. Biopharm. 2004, 58, 409.

17. Guilhermea, M.R.; da Silvaa, R.; Rubiraa, A.F.; Geuskensb, G.; Muniza, E.C. React. Funct. Polym. 2004, 61, 233.

18. Lie-Wen, X.; Xiao-Jie, J.; Jing-Jing, L.; Rui, X.; Liang-Yin, C. J. Coll. Interf. Sci. 2010, $349,106$.

19. Gundogan, N.; Melekaslan, D.; Okay, O. Eur. Polym. J. 2003, 39, 2209.

20. Krusic, M.K.; Filipovic, J. Polym. 2006, 47, 148.

21. Xu, X.D.; Zhang, X.Z.; Cheng, S.X.; Zhuo, R.X.; Kennedy, J.F. Carbohyd. Polym. 2007, 68, 416.

22. Y1ldı, B.; Isık, B.; K1s, M. Eur. Polym. J. 2002, 38, 1343.

23. Jones, D.S.; Brown, A.F.; Woolfson, A.D. J. Appl. Polym. Sci. 2003, 87, 1016.

24. Aixiang, Q.; Mangeng, L.; Qunfeng, L.; Ping, Z. Front. Chem.China 2007, 2, 135.

25. Erbil, C.; Kazancioglu, E.; Uyanık, N. Eur. Polym. J. 2004, 40, 1145.

26. Freitas, R.F.S.; Sousa, G.R.; Magalhaes, W.F. Polym. Degrad. Stab. 1998, 61, 274.

27. Huang, Y.; Yu, H.; Xiao, C. Carbohyd. Polym. 2007, 69, 774.

28. Hirokawa,Y.; Tanaka, T. J. Chem. Phys. 1984, 81, 6379.

29. Tokuhiro, T.; Aniya, T.; Manada, A.; Tanaka, T. Macromol. 1991, 24, 2936.

30. Hertle, Y.; Zeiser, M.; Hasenoehrl, C.; Busch, P.; Hellweg, T. Coll. Polym. Sci. 2010, 288, 1047.

31. Yildiz, B.; Isik, B.; Kis, M. React. Funct. Polym. 2002, 52, 3.

32. Park, T.G.; Hoffman, A.S. Biotechnol. Prog. 1994, 10, 82.

33. Tasdelen, B.; Kayaman Apohan, N.; Guven, O.; Baysal, B.M. Rad. Phys. Chem. 2005, 73, 340.

34. Shibayama, M.; Fujikawa, Y.; Nomura, S. Macromol. 1996, 29, 6535.

35. Vernon, B.; Kim, S.W.; Bae, Y.H. J. Biomed. Mater. Res. 2000, 51, 69. 36. Park, T.G.; Hoffman, A.S. J. Biomed. Mater. Res. 1990, 24, 21. 